\title{
First evidence of multiple populations along the AGB from Strömgren photometry $\star, \star \star$
}

\author{
Pieter Gruyters ${ }^{1}$, Luca Casagrande ${ }^{2}$, Antonino P. Milone ${ }^{2}$, Simon T. Hodgkin ${ }^{3}$, Aldo Serenelli ${ }^{4}$, and Sofia Feltzing ${ }^{1}$ \\ ${ }^{1}$ Lund Observatory, Department of Astronomy and Theoretical Physics, Box 43, 22100 Lund, Sweden \\ e-mail: pieter.gruyters@physics.uu.se \\ 2 Research School of Astronomy and Astrophysics, Mount Stromlo Observatory, The Australian National University, ACT 2611, \\ Australia \\ 3 Institute of Astronomy, Madingley Road, Cambridge CB3 OHA, UK \\ ${ }^{4}$ Institute of Space Sciences (IEEC-CSIC), Carrer de Can Magrans S/N, 08193 Barcelona, Spain
}

Received 23 December 2016 / Accepted 19 April 2017

\begin{abstract}
Spectroscopic studies have demonstrated that nearly all Galactic globular clusters (GCs) harbour multiple stellar populations with different chemical compositions. Moreover, colour-magnitude diagrams based exclusively on Strömgrem photometry have allowed us to identify and characterise multiple populations along the RGB of a large number of clusters. In this paper we show for the first time that Strömgren photometry is also very efficient at identifying multiple populations along the AGB, and demonstrate that the AGB of M 3, M 92, NGC 362, NGC 1851, and NGC 6752 are not consistent with a single stellar population. We also provide a catalogue of RGB and AGB stars photometrically identified in these clusters for further spectroscopic follow-up studies. We combined photometry and elemental abundances from the literature for RGB and AGB stars in NGC 6752 where the presence of multiple populations along the AGB has been widely debated. We find that, while the MS, SGB, and RGB host three stellar populations with different helium and light element abundances, only two populations of AGB stars are present in the cluster. These results are consistent with standard evolutionary theory.
\end{abstract}

Key words. Hertzsprung-Russell and C-M diagrams - stars: abundances - techniques: photometric - globular clusters: general

\section{Introduction}

Nearly all the old globular clusters (GCs) are characterised by star-to-star variations in the light elements, including $\mathrm{He}$, C, N, O, and Na (e.g. Milone et al. 2014; Pancino et al. 2010; Carretta et al. 2009a). Moreover, some clusters exhibit variations in $\mathrm{Li}, \mathrm{Mg}, \mathrm{Al}$, and Si (e.g. D'Orazi et al. 2015; Carretta et al. 2009b; Gruyters et al. 2014). Some massive "anomalous" clusters also have internal variations in some of the heavy elements (e.g. Marino et al. 2015); see Gratton et al. (2012) for a review on the chemical composition of multiple populations in GCs.

The origin of multiple stellar populations in GCs is still not fully understood. Some authors have concluded that GCs have experienced multiple episodes of star formation during which a second generation (SG) of stars is formed from material polluted by processed material from massive first generation (FG) stars (e.g. D'Antona et al. 2002; Decressin et al. 2007; Denissenkov \& Hartwick 2014).

Other groups suggest that the chemical variations are due to accretion of polluted material in the pre-main sequence phase (Bastian et al. 2013); Renzini et al. (2015), Bastian (2015), and

\footnotetext{
* Based on observations made with the Isaac Newton Telescope operated on the island of La Palma by the Isaac Newton Group in the Spanish Observatorio del Roque de los Muchachos of the Instituto de Astrofísica de Canarias.

$\star \star$ Full Tables B.1 and B.2 are only available at the CDS via anonymous ftp to cdsarc.u-strasbg. fr (130.79.128.5) or via http://cdsarc.u-strasbg.fr/viz-bin/qcat?J/A+A/603/A37
}

D'Antona et al. (2016) provide critical discussions of the different scenarios.

Photometry is a powerful tool to identify multiple stellar populations. Multi-wavelength photometry from the Hubble Space Telescope (HST) has revealed multiple main sequences (MSs), sub-giant branches (SGBs) and red giant branches (RGBs) in nearly all the analysed GCs (see e.g. Piotto et al. 2015; Milone et al. 2017, and references therein). These multiple sequences correspond to stellar populations with different helium and light elements abundances and strongly affect the morphology of the colour-magnitude diagram (CMD) of star clusters. Recent papers have provided direct evidence that even the shape of the horizontal branch is closely connected with the presence of multiple populations (e.g. Marino et al. 2011, 2014a; Gratton et al. 2012, and references therein) .

Multiple RGBs have also been identified using groundbased photometry (e.g. Marino et al. 2008; Monelli et al. 2013; Massari et al. 2016) as demonstrated by the pioneering papers by Frank Grundahl and his collaborators based on Strömgren photometry (e.g. Grundahl et al. 1998, 1999; Yong et al. 2008; Carretta et al. 2011a).

The asymptotic giant branch (AGB) has been poorly investigated in the context of multiple populations. Nevertheless, the presence (or the absence) of stellar populations along the AGB is crucial to constrain the models of stellar evolution. Indeed helium-rich stars in GCs, which have lower mass than stars with primordial helium abundance during the horizontal branch (HB) phase, can evolve into so-called AGB manqué 
stars that never reach the AGB (e.g. Sweigart \& Gross 1976; Dorman et al. 1993).

Early spectroscopic studies by Mallia (1978), Norris et al. (1981), Smith \& Norris (1993), and more recently Campbell et al. (2006) report a possible lack of CN-strong stars along the AGB and therefore hint that second-population stars in GCs avoid the AGB phase. In contrast, recent studies by, for example Ivans et al. (2001), Johnson et al. (2015), and Wang et al. (2016) have revealed that the AGB of some clusters, such as NGC 5904 and 47 Tuc, exhibit star-to-star light element variations, in close analogy with what has been observed along their RGBs.

NGC 6752 is an intriguing case and has been strongly debated in recent papers. Photometry and spectroscopy of RGB and MS stars have revealed that this cluster hosts three stellar populations with different content of helium and light elements and that only $\sim 30 \%$ of its stars belong to a population of FG stars with primordial helium abundance (Yong et al. 2013; Milone et al. 2013, 2017; Dotter et al. 2015); see also Carretta et al. (2007) for the study of the sodium-oxygen anti-correlation along the RGB of NGC 6752. Intriguingly, Campbell et al. (2013) derived $\mathrm{Na}$ abundances for $24 \mathrm{RGB}$ and 20 AGB stars and from the $[\mathrm{Na} / \mathrm{Fe}]$ distribution they concluded that there are no SG AGB stars with enhanced $\mathrm{Na}$ and $\mathrm{He}$ (i.e. 2nd and 3rd population in NGC 6752, Milone et al. 2013) and that only $\sim 30 \%$ of NGC 6752 stars undergo the AGB phase. This fact would suggest that the majority of NGC 6752 stars enhanced in He (i.e. SG stars) do not ascend the AGB (see discussion by Cassisi et al. 2014). Noticeably, NGC 6752 hosts a very extended $\mathrm{HB}$ which is well populated on the blue side of the Grundahl et al. (1999) jump (e.g. Brown et al. 2016). Campbell and collaborators suggested that the metal enhancement due to radiative levitation of $\mathrm{HB}$ stars hotter than $\sim 11500 \mathrm{~K}$ may cause enhanced mass loss and push HB stars into the AGB-manqué phase. This conclusion was challenged by Lapenna et al. (2016) who re-observed the $20 \mathrm{AGB}$ stars and derived $\mathrm{Na}$ and $\mathrm{O}$ abundances amongst other. Based on their derived abundances Lapenna et al. (2016) conclude that SG AGB stars with enhanced $\mathrm{Na}$ are also present (see also Cassisi et al. 2014).

Interestingly, the spectroscopic investigation of 6 AGB stars and 13 RGB stars in M62 suggests that its second-generation stars avoid the AGB phase (Lapenna et al. 2016). This cluster exhibits a very extended $\mathrm{HB}$ and about $80 \%$ of its stars are highly helium enhanced by $\Delta Y \sim 0.08$ with respect to the primordial value (Milone 2015). These observations are consistent with the scenario where AGB-manqué stars have high helium abundance (e.g. Gratton et al. 2010).

The GC M4 is another controversial case. This cluster hosts two main stellar populations with different oxygen and sodium abundance, which correspond to distinct photometric sequences along the RGB and the MS (e.g. Marino et al. 2008; Milone et al. 2014). The sodium-rich stellar population is only slightly enhanced in helium with respect to the sodium poor population by $\Delta Y \sim 0.02$ (e.g. Villanova et al. 2012; Nardiello et al. 2015).

In addition, the $\mathrm{HB}$ of M 4 is well populated on both sides of the RR Lyrae instability strip but its stars never exceed $\sim 9000 \mathrm{~K}$ and are not affected by radiative levitation. Moreover, Marino et al. (2011) and Villanova et al. (2012) found that the blue HB stars are sodium rich and oxygen poor, while the red HB stars have the same chemical composition as the first populations.

In this context, the conclusion of a recent spectroscopic study of AGB stars that second-population stars of M 4 may avoid the
AGB phase (MacLean et al. 2016) challenges the prediction of standard stellar evolution theory. Indeed we would expect that the blue HB stars of M4, which are only marginally helium enhanced and belong to the blue $\mathrm{HB}$, would evolve into the AGB. Noticeably, the result by MacLean and collaborators is not confirmed by the successive work by Lardo et al. (2017) who have analysed $U, B, V, I$ photometry and have found that the AGB of M4 is not consistent with a simple population.

In this paper we study for the first time the AGB of five GCs, namely M 3, M 92, NGC 362, NGC 1851, and NGC 6752 using Strömgren photometry. We are in particular interested in revealing the presence of multiple populations along the AGB in these clusters. It has been shown that CMDs built with the appropriate combination of ultraviolet and optical HST filters are able to identify multiple populations along the AGB (e.g. Milone et al. 2015b,a; Nardiello et al. 2015, for NGC 2808, NGC 7089, and NGC 6352). Multiple sequences of AGB stars have also been detected with a combination of wide-band ground-based photometry (e.g Monelli et al. 2013; García-Hernández et al. 2015; Lardo et al. 2017). Here we leverage on the combination of Strömgren filters to achieve the same goal. In fact, it has already been shown that Strömgren photometry is very efficient in identifying stellar populations with different chemical compositions (e.g. Grundahl et al. 1998; Yong et al. 2008).

We also combine information from the spectroscopic analysis by Campbell et al. (2013) and Lapenna et al. (2016) and multi-wavelength Strömgrem photometry to investigate multiple populations along the AGB of NGC 6752 in detail.

\section{Observations}

The analysis presented in this paper is based on Strömgren photometry. We combine our own data obtained during the Stömgren survey for Asteroseismology and Galactic Archeology (SAGA; Casagrande et al. 2014) and literature data collected by Grundahl and co-workers. The Strömgren uvby system (Strömgren 1963) was developed to obtain basic stellar parameters such as effective temperature, surface gravity, and metallicity (see e.g. Árnadóttir et al. 2010, and references therein).

\subsection{SAGA data}

The SAGA survey (Casagrande et al. 2014) is based on observations collected with the Wide Field Camera (WFC) at the Isaac Newton Telescope (INT). Although the main goal of SAGA is to obtain Strömgren photometry of stars in the Kepler and K2 fields, a number of open and globular clusters are also targeted. Here we used photometry obtained in June 2012 for the globular cluster M 3. For the data reduction we used an update of the procedure described in Casagrande et al. (2014) and derived PSF photometry with the method and computer programs by Anderson \& King (2006) and Anderson et al. (2006) adapted to the INT/WFC camera.

\subsection{Literature data}

We used the photometry collected by Grundahl and co-workers and presented by Calamida et al. (2007) ${ }^{1}$. The data consists of fully calibrated PSF and aperture photometry. Information concerning the observations, data reduction and calibration procedures can be found in Grundahl et al. (1999, 2002). We use

\footnotetext{
1 The catalogues were downloaded from the web page http://www. oa-roma . inaf.it/spress/gclusters.html
} 
the data for four GCs, i.e. M92, NGC 362, NGC 1851, and NGC 6752.

\section{Analysis}

In this section we analyse the available dataset to investigate multiple stellar populations of five clusters along the AGB and the RGB. In the following subsection we use the example of NGC 6752 to demonstrate that Strömgren photometry can be used to identify multiple populations along the AGB and RGB, while in the Sect. 3.2 we extend the analysis to M3, M92, NGC 362, and NGC 1851.

\subsection{Multiple stellar populations in NGC 6752}

The identification and characterisation of multiple populations in the CMD requires accurate photometry. To obtain the best possible cluster photometry, we have identified a sample of wellmeasured GC stars, according to three selection criteria, and we limited our analysis to the selected stars.

- We first rejected stars in the central regions of each cluster where the photometry has a poorer quality due to stellar crowding. Specifically, we rejected stars with radial distance from the cluster centre, $R<45$ arcsec in the case of M 3 and stars with $R<90$ arcsec in the remaining clusters.

- We selected stars with relatively small photometric errors, which have been estimated as the ratio between the rms of the $N$ independent magnitude measurements divided by the square root of $N-1\left(N \sim 10^{4}-10^{5}\right)$. Specifically, we rejected all the stars with an error larger than $e r r_{\text {med }}+0.015$, where $e r r_{\text {med }}$ is the median error of stars with similar magnitude (typically $u_{\text {err }}<0.01$ for $y<16$ ).

- Moreover, we included in our analysis only stars within a narrow $m_{1}=(v-b)-(b-y)$ interval. As the Strömgren $m_{1}$ index is primarily sensitive to metallicity, this criterium ensures that we mostly selected evolved stars with the same metallicity as the cluster and we minimised contamination by field stars.

The $(b, b-y)$ and $(y, v-y)$ CMDs of the selected NGC 6752 stars are shown in Fig. 1. We constructed fiducial sequences for the RGB and AGB by eye in such a way that they pass through the AGB and RGB stars selected by Campbell et al. (2013). For the other clusters we used fiducial sequences to identify a sample of AGB and RGB stars that are located within a certain distance from these fiducial lines. Details on the selection rules are provided in Table A.1.

The $c_{\mathrm{y}}=(u-v)-(v-y)$ index was defined by Yong et al. (2008) as a colour index that is sensitive to the stellar nitrogen abundance but almost insensitive to temperature. Owing to the $\mathrm{N}$-sensitivity, the $c_{\mathrm{y}}$ index can effectively distinguish between stellar populations with different light element content. The left panel of Fig. 2 shows the $\left(b, c_{\mathrm{y}}\right)$ diagram for the selected AGB and RGB stars. The typical observational errors are indicated by the error bars plotted on the left of this diagram. We immediately note that the $c_{\mathrm{y}}$ spread of both AGB and RGB stars is much larger than what we expect from observational errors alone. To quantify this phenomenon, we determined the spread in the $c_{\mathrm{y}}$ index on the AGB and RGB by constructing an RGB and AGB fiducial sequence in the $\left(y, c_{\mathrm{y}}\right) \mathrm{CMD}$ and calculating the standard deviation around these sequences. We found that the spread in the $c_{\mathrm{y}}$ index on the AGB and RGB in NGC 6752 is $~ 0.05$ mag for AGB and RGB, respectively, and is significantly larger than the spread stemming from the observational error, which is $\sim 0.01 \mathrm{mag}$.
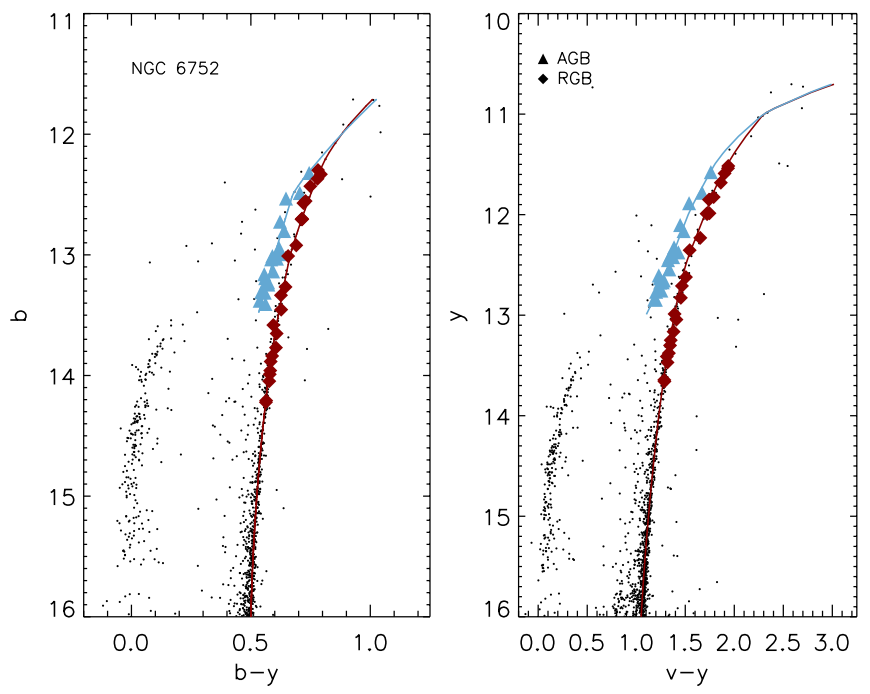

Fig. 1. Colour-Magnitude diagrams of $(b, b-y)(l e f t)$ and $(y, v-y)(r i g h t)$ for NGC 6752. The RGB and AGB stars studied by Campbell et al. (2013) are denoted with diamonds and triangles, respectively. The blue and brown lines are the fiducial sequences of the AGB and the RGB, respectively.
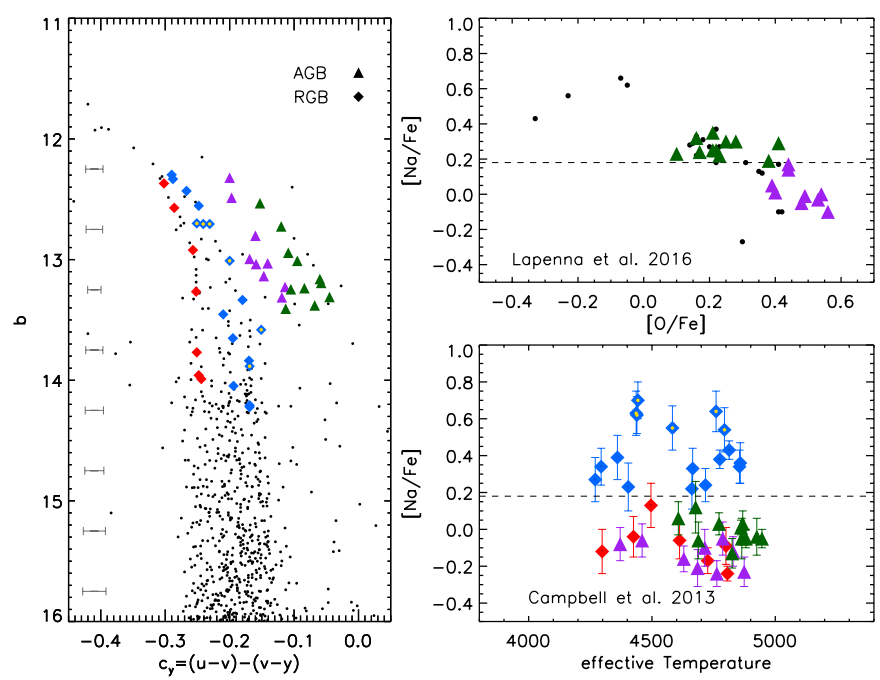

Fig. 2. Diagram of $\left(b, c_{\mathrm{y}}\right)$ for AGB and RGB stars of NGC 6752 (left). The typical photometric errors are indicated by the error bars on the left of the diagram. The upper right panel compares the sodium-oxygen anti-correlation for RGB stars (bullets) and AGB stars (triangles) from Yong et al. (2013) and Lapenna et al. (2016). The dashed line separates population A and B AGB stars, which are coloured green and purple, respectively. The same colours are used to represent these AGB stars in the other panels of this figure. The lower right panel shows $[\mathrm{Na} / \mathrm{Fe}]$ as a function of the stellar effective temperature for RGB (diamonds) and AGB stars (triangles) from Campbell et al. (2013). The dashed line separates the two groups first and second population stars defined by Campbell and collaborators, which are coloured red and blue, respectively. RGB stars with $[\mathrm{Na} / \mathrm{Fe}]>0.5$ are denoted with yellow dots. These symbols and colours are used to represent the same RGB stars in the diagram in the left panel.

The fact that the RGB is intrinsically broadened in the $c_{\mathrm{y}}$ index reveals that NGC 6752 hosts multiple populations (see e.g. Yong et al. 2008). Indeed the $c_{\mathrm{y}}$ index is strongly sensitive to the nitrogen abundance of the different stellar populations and the $c_{\mathrm{y}}$ value of an RGB star strongly correlates with its nitrogen 
abundance (see Fig. 7 from Yong et al. 2008, for an example). Similarly, the $c_{\mathrm{y}}$ broadening of AGB stars demonstrates that the AGB is not consistent with a simple population.

To further investigate stellar populations along the AGB and RGB of NGC 6752 we combine photometry with elemental abundances from the literature. In the upper right panel of Fig. 2 we reproduce the sodium-oxygen anti-correlation for AGB stars (coloured triangles; Lapenna et al. 2016) and RGB stars (black bullets; Yong et al. 2013).

The RGB of NGC 6752 hosts three main populations with different $\mathrm{O}, \mathrm{Na}$, and helium: the two stellar groups with a helium fraction $Y \sim 0.25-0.26$ are clustered around $[\mathrm{O} / \mathrm{Fe}] \sim 0.4$ and $[\mathrm{O} / \mathrm{Fe}] \sim 0.2$ in the $[\mathrm{Na} / \mathrm{Fe}]$ versus $[\mathrm{O} / \mathrm{Fe}]$ plane. The third group of stars has extreme oxygen and sodium abundances and has high helium abundances $Y \sim 0.27-0.28$ (Milone et al. 2013; Dotter et al. 2015). Milone et al. (2013) called the stellar group with high helium content population $\mathrm{C}$, while the other two populations with $Y \sim 0.25$ and $\sim 0.26$ are indicated as $\mathrm{A}$ and $\mathrm{B}$, respectively. For clearness, in the following we adopt the terminology by Milone and collaborators. Since stars of the same age but different helium abundances have different masses, their presence or absence along the AGB are crucial to constrain stellar evolution models.

The RGB and AGB of NGC 6752 have been studied spectroscopically by Campbell et al. (2013) who discovered that AGB stars span a smaller range of Na than RGB stars. These authors concluded that all second-population stars avoid the AGB phase and invoked extreme mass loss in second-population HB stars. Specifically, they suggested that these stars are located on the blue side of the Grundahl et al. (1999) jump and that their mass loss is enhanced by the effect of metal enhancement associated with radiative levitation. Campbell and collaborators did not account for the three stellar populations A, B, and C of NGC 6752, but adopted a simple scheme whereby the analysed stars only include a first population with primordial chemical composition and a second population of stars enhanced in helium and sodium.

Lapenna et al. (2016) analysed $\mathrm{Na}$ and $\mathrm{O}$ of the same AGB stars in NGC 6752 that were already analysed by Campbell et al. (2013) and discovered that the AGB hosts stars with different sodium and oxygen abundances, thus demonstrating that the AGB is not consistent with a simple population. The two populations of AGB stars with primordial and intermediate chemical composition, correspond to the populations A and B and have low helium abundance $(Y \lesssim 0.26)$.

Noticeably, Lapenna and collaborators confirmed that the stars with high sodium (hence low oxygen) abundance, are not present along the AGB. These stars belong to the helium-rich population $\mathrm{C}$ identified photometrically.

Both populations A and B of AGB stars are clearly visible in the upper right panel of Fig. 2 and are coloured purple and green, respectively. The same colours are used to represent the same stars in the $\left(b, c_{\mathrm{y}}\right)$ diagram of Fig. 2.

Population A and population B stars define almost distinct sequences and population B AGB stars have larger $c_{\mathrm{y}}$ values than population A AGB stars with the same luminosity. This fact confirms that the $c_{\mathrm{y}}$ broadening of AGB stars is actually due to the presence of multiple stellar populations of AGB stars and demonstrates that Strömgren photometry is very efficient in identifying multiple populations along the AGB.

In the lower right panel of Fig. 2 we show $[\mathrm{Na} / \mathrm{Fe}]$ versus $T_{\text {eff }}$ for AGB stars (triangles) and RGB stars (diamonds) from Campbell et al. (2013). Purple and green colours indicate the AGB stars with primordial and intermediate chemical composition identified in the upper right panel of Fig. 2. These
Table 1. Spread in the $c_{\mathrm{y}}$ index and average $c_{\mathrm{y}}$ uncertainty due to photometric errors.

\begin{tabular}{lccc}
\hline \hline GC & $\begin{array}{c}c_{\mathrm{y}} \text { spread } \\
\text { RGB }\end{array}$ & $\begin{array}{c}c_{\mathrm{y}} \text { spread } \\
\text { AGB }\end{array}$ & $c_{\mathrm{y}}$ unc \\
\hline NGC 6752 & 0.05 & 0.05 & 0.01 \\
NGC 1851 & 0.03 & 0.05 & 0.01 \\
NGC 362 & 0.02 & 0.03 & 0.01 \\
M 92 & 0.02 & 0.02 & 0.01 \\
M 3 & 0.04 & 0.05 & 0.02 \\
\hline
\end{tabular}

two groups of stars have, on average, different sodium abundances thus demonstrating that the group of stars that, for simplicity, has been indicated as a first population by Campbell et al. (2013) actually include two groups of stars (A and B) with slightly different helium abundance.

For completeness, we identify in the $[\mathrm{Na} / \mathrm{Fe}]$ versus $T_{\text {eff }}$ plane three groups of RGB stars with different sodium abundances and indicate these with red, blue, and yellow bullets within blue diamonds in Fig. 2. We confirm that stars with different sodium abundances populate different RGB regions in the $\left(b, c_{\mathrm{y}}\right)$ plane as previously noticed by Yong et al. (2008). Noticeably, stars with extreme $\mathrm{Na}$ and $\mathrm{O}$ abundances are not present near the RGB tip. The small number of stars prevents us from making a strong conclusion and further spectroscopic investigation of more stars is needed to determine whether this phenomenon is intrinsic or if it is due to small statistics.

\subsection{Multiple stellar populations along the AGB of M3, M92, NGC 362, and NGC 1851}

Having demonstrated that the $c_{\mathrm{y}}$ provides an efficient way to identify multiple populations along the AGB, we extend the analysis to four other clusters, namely M3, M92, NGC 362, and NGC 1851.

The results are shown in Fig. 3. The figure shows the $\left(c_{\mathrm{y}}, b\right)$ CMDs of the clusters along with histograms of the spread in $c_{\mathrm{y}}$ on the RGB (top panel) and AGB (middle panel) as well as the uncertainty distribution based on the photometric uncertainties in $c_{\mathrm{y}}$ (bottom panel). To obtain the spread in $c_{\mathrm{y}}$ we construct an RGB and an AGB fiducial sequence (given by the lines in the CMDs) in the $\left(c_{\mathrm{y}}, b\right)$ CMD. The actual $c_{\mathrm{y}}$ spread is then the standard deviation $\sigma$ around the fiducial sequence. The typical photometric uncertainty on the $c_{\mathrm{y}}$ index for the RGB and AGB is also given by the error bar on the right side of the CMDs. The spreads are summarised in Table 1 along with the average photometric uncertainties in $c_{\mathrm{y}}$.

Comparing the uncertainty distributions with the spread on the RGB/AGB shows immediately that the spread in $c_{\mathrm{y}}$ is real and not the result of the photometric uncertainties. As all clusters have spectroscopically confirmed multiple populations (albeit not on the AGB), we conclude that the spread on the RGB and the AGB is due to the presence of multiple populations.

The fact that the AGB of M3 is not consistent with a simple stellar population confirms previous findings by García-Hernández et al. (2015). These authors used APOGEE spectroscopy to show that M3 second-generation AGB stars with enhanced aluminium abundance. Moreover, the wide spread in that they observed in the $V$ versus $C_{\mathrm{U}, \mathrm{B}, \mathrm{I}}$ further demonstrates that M 3 hosts multiple populations of AGB stars. 
P. Gruyters et al.: First evidence of multiple populations along the AGB from Strömgren photometry
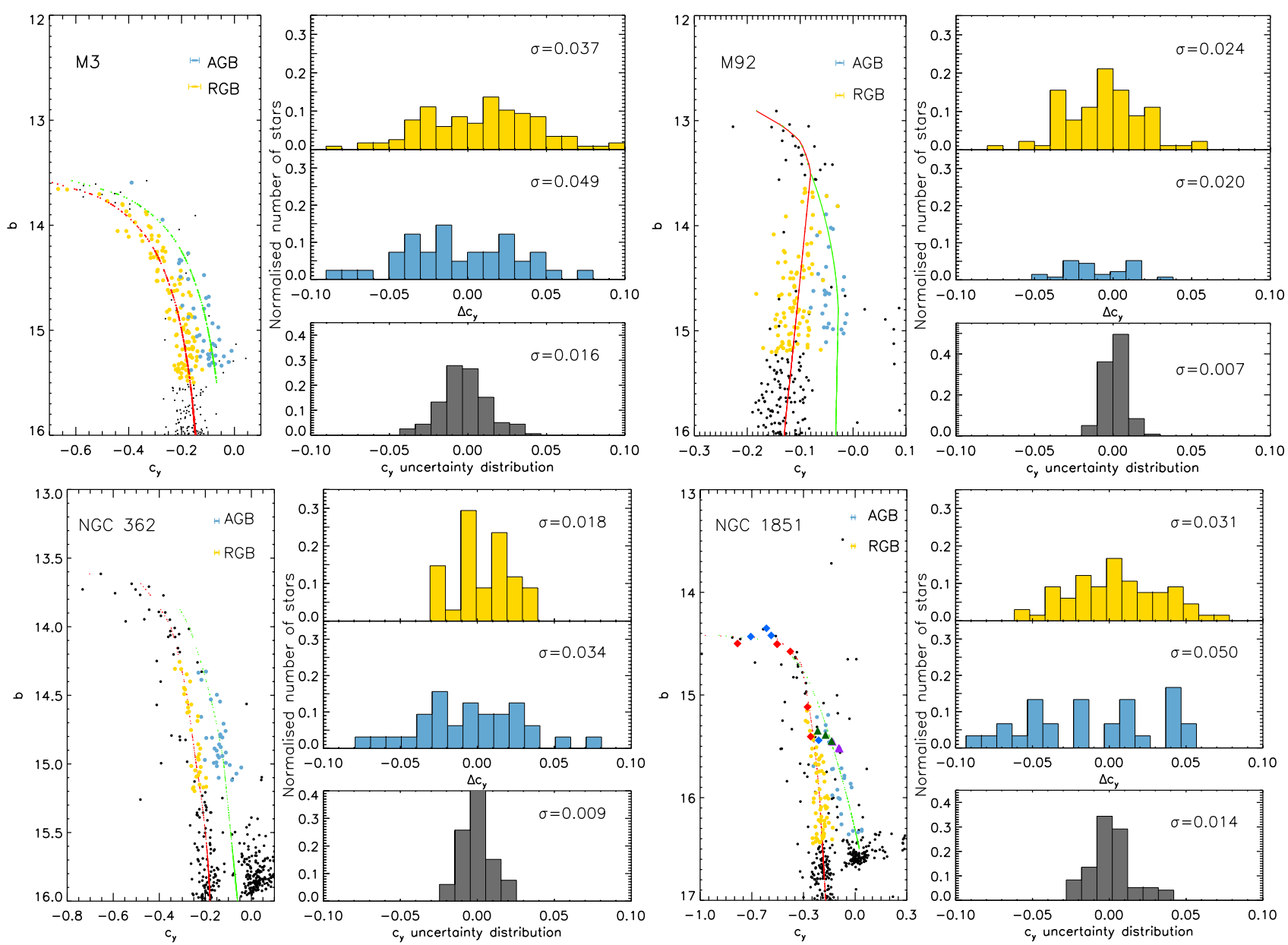

Fig. 3. Colour-magnitude diagrams of $b$ vs. $c_{\mathrm{y}}$ and $c_{\mathrm{y}}$ spreads for M 3 (top left), M 92 (top right), NGC 362 (bottom left), and NGC 1851 (bottom right). In all plots the RGB stars are given by the yellow bullets, while the blue bullets represent the AGB stars. The typical photometric uncertainty for each group is given by the error bars in the upper right part of the CMDs (yellow for RGB and blue for AGB) and the bottom histogram in each plot shows the distribution of the photometric uncertainties in $c_{\mathrm{y}}$. The fiducial sequences for the RGB and AGB are also plotted in the CMDs. The coloured histograms show the spread around these fiducial sequences. The standard deviation $\sigma$ is also given. Abundance information for some of the stars in NGC 1851 is overplotted in the bottom right CMD, where navy blue and purple indicate FG stars and red and green indicate SG stars based on the $\mathrm{CNO}$ and $\mathrm{Na}$ abundances available in the literature (see text).

\section{Discussion}

For the first time, we showed the presence of multiple populations along the RGB and AGB based on Strömgren photometry. Figure 3 shows that the $c_{\mathrm{y}}$ spread is similar on the AGB and RGB for all clusters.

The $c_{\mathrm{y}}$ spread in NGC 6752 is the largest in our sample of clusters (see Table 1). The large spread in $c_{\mathrm{y}}$ is expected as NGC 6752 shows a large abundance spread in the light elements (e.g. 1 dex in N, 0.8 dex in $\mathrm{Na}$ and $\mathrm{O}, 0.5$ dex in $\mathrm{Mg}$ and 1.5 dex in $\mathrm{Al}$ Yong et al. 2005, 2008, 2013). For the other clusters the $c_{\mathrm{y}}$ spread varies from 0.018 to $0.037 \mathrm{mag}$ on the RGB and 0.020 to 0.050 on the AGB. Three out of five clusters show the same $c_{\mathrm{y}}$ spreads, within the errors, on the RGB and AGB. The $c_{\mathrm{y}}$ spread is different on the AGB compared to the RGB for NGC 362 and NGC 1851. This could be due to the smaller sample size compared to the RGB sample. As we have fewer data points on the AGB, the data becomes more susceptible to outliers. The fact that the $c_{\mathrm{y}}$ spread is significant compared to the photometric errors and that all clusters have confirmed multiple populations, tells us that the $c_{\mathrm{y}}$ spread is due to multiple populations. We conclude that the AGBs in the four clusters under investigation are populated by FG and SG stars. One can thus select FG and
SG AGB stars based on their location in the $\left(c_{\mathrm{y}}, b\right)$ plane where the FG stars are located on the left side of the RGB and AGB and SG are located on the right side.

Aside for NGC 6752, we were also able to find three sets of C, N, O, and Na abundances in NGC 1851, one set for four RGB stars (Yong et al. 2015), a second set for four AGB and five RGB stars (Yong et al. 2009) and a third set for two AGB stars from the ESO Phase 3 archive system ${ }^{2}$. Comparing these abundances within each sample we identify two populations based on whether or not the $\mathrm{N}$ and/or $\mathrm{Na}$ abundance are low compared to the sample maximum. We have colour-coded these stars in the bottom right panel of Fig. 3 where blue and purple symbols identify stars low in $\mathrm{N}$ and $\mathrm{Na}$ and red and green symbols stars high in $\mathrm{N}$ and $\mathrm{Na}$. Two notes should be made with respect to NGC 1851:

1) While the majority of GCs have homogeneous s-process elements and $\mathrm{C}+\mathrm{N}+\mathrm{O}$ abundance, NGC 1851 hosts two main populations: one anomalous population enriched in $\mathrm{C}+\mathrm{N}+\mathrm{O}$ and s-process elements and one so-called normal population characterised by solar s-process elements (Yong et al.

2 http://archive.eso.org/cms.html 
2009, 2015; Carretta et al. 2011b; Marino et al. 2014b) ${ }^{3}$. Intriguingly, both s-rich and s-normal stars are not consistent with a simple population and host sub-populations with different light element abundances (e.g. Lardo et al. 2012; Marino et al. 2014b), where s-rich stars have, on average, higher $\mathrm{Na}$ and $\mathrm{N}$ content than s-normal stars (e.g. Yong et al. 2008; Lardo et al. 2012). We here identify two stellar groups based on the $\mathrm{N}$ and $\mathrm{Na}$ abundances. The identified stars can thus belong to the two main populations in NGC 1851 and/or to subpopulations making up the normal population.

2) The middle part of the AGB shows a clear split, possibly as a result of multiple populations. Interestingly we find that the stars to the right of the AGB fiducial (purple triangles) have solar barium abundances. We thus seem to find that the right side of the AGB is populated by the normal population. Unfortunately, we did not find any information on $\mathrm{Ba}$ for stars on the left side of the AGB fiducial and hence we cannot conclude that the left side of the AGB is populated by the anomalous population. It would however, be interesting to see whether the double AGB is due to abundance differences in $\mathrm{C}+\mathrm{N}+\mathrm{O}$ and s-process elements, and/or FG/SG stars with only differences in the abundances of light elements. We therefore provide a catalogue (see Table B.1) of the identified AGB and RGB stars for this and the three other clusters in the hope that they will be useful when selecting AGB and RGB stars for future spectroscopic analyses.

We now turn back to NGC 6752 and compare the observations with stellar evolution theory. From stellar evolution theory we expect that not all stars reach the AGB, in particular horizontal branch stars with $M \lesssim 0.5-0.6 M_{\odot}$ do not ascent the AGB (Gratton et al. 2010). According to Milone et al. (2013), NGC 6752 is characterised by three chemical populations, a FG that is made up of stars with normal $\mathrm{He}$ and low $\mathrm{Na}(Y \sim 0.25$, $[\mathrm{Na} / \mathrm{Fe}] \sim-0.03)$, and a $\mathrm{SG}$ that is made up of stars enhanced in $\mathrm{He}$ and $\mathrm{Na}$. This $\mathrm{SG}$ can be split up into stars with a normal $\mathrm{He}$ and $\mathrm{Na}$ enhancement (intermediate population, $\Delta Y=0.01$ and $[\mathrm{Na} / \mathrm{Fe}] \sim 0.26$ ) and stars strongly enhanced in $\mathrm{He}$ and $\mathrm{Na}$ (extreme population, $\Delta Y=0.03$ and $[\mathrm{Na} / \mathrm{Fe}] \sim 0.61$ ). From the observed abundances we see that stars of the extreme stellar population with Y 0.28-29 do not ascend the AGB, while stars in the remaining two populations $(Y \sim 0.25$ and $Y \sim 0.26)$ are present along the AGB. This finding is completely in line with Lapenna et al. (2016) but also shows that Campbell et al. (2013) was correct in stating that stars with $Y=0.285$ do not reach the AGB.

The observations are in accordance with the synthetic HB simulations by Cassisi et al. (2014), which considers three stellar populations with $Y=0.24,0.25$ and 0.27 to reproduce the observed AGB-to-RGB ratio, HB morphology, HB magnitude, and $\mathrm{HB}$ colour distribution without invoking exceptional mass loss during the HB phase. The behaviour can be explained as follows.

\footnotetext{
The presence of star-to-star variations in the overall $\mathrm{C}+\mathrm{N}+\mathrm{O}$ abundance in NGC 1851 has been challenged by Villanova et al. (2010) who have analysed spectra of this cluster and concluded that NGC 1851 has homogeneous $[(\mathrm{C}+\mathrm{N}+\mathrm{O}) / \mathrm{Fe}]$. However, the conclusion by Villanova et al. (2010) is in contrast with photometric observations of NGC 1851 (Milone et al. 2008, 2017; Han et al. 2009; Lardo et al. 2012). Although Milone et al. (2008) showed that the splitting of the SGB can be explained with an age difference of about $1 \mathrm{Gyr}$, the most likely explanation is that the split SGB and RGB in the CMD are the result of a second population with enhanced $\mathrm{C}+\mathrm{N}+\mathrm{O}$ (e.g. Sbordone et al. 2011; Cassisi et al. 2008; Ventura et al. 2009).
}

According to the outline in Gratton et al. (2010), horizontal branch stars with mass $M \lesssim 0.5-0.6 M_{\odot}$ do not ascend the AGB. These so-called AGB-manqué stars (Greggio \& Renzini 1990) have been found in several clusters (see e.g. the case of NGC 2808: Castellani et al. 2006). Similarly, if, for a given value of the initial He abundance, the mass along the RGB is efficient enough to reduce the mass of the star below the critical value for the He-core flash, the He-flash does not occur. Subsequently, the star leaves the RGB before reaching its tip and populates the blue hook of the HB before populating the white-dwarf cooling sequence ${ }^{4}$. Hence these stars are called RGB-manqué and do not ascend the AGB either.

Some indications of this outline can perhaps be noted when looking at the data for NGC 6752. We have marked the extreme population on the RGB in Fig. 2 with a small yellow bullet within the blue diamonds giving the Na-rich stars in NGC 6752. Interestingly, it seems that the tip of the RGB is not populated with extreme stars. Likewise on the AGB, no intermediate SG stars seem to to reach luminosities as high as FG on the AGB. It is possible that this is just a result of small number statistics. Nevertheless we would urge the spectroscopic community to investigate this further as the spectroscopic information can be used to test stellar evolution predictions, which tell us that the extreme/intermediate population produces AGB/RGB-manqué objects.

Several works showed that the position of a star along the $\mathrm{HB}$ depends on its mass and that the mass in turn is correlated with the chemical composition through the difference in He abundance amongst the different populations (e.g. D'Antona et al 2002; Marino et al. 2011). It is then expected that some Herich $\mathrm{HB}$ stars, which are extreme SG stars, do not reach the AGB while SG HB stars with less-extreme chemical compositions can reach the AGB. In some cases, SG stars can terminate their AGB evolution before reaching very bright luminosities (e.g. Cassisi et al. 2014, and references therein). This scenario is consistent with our results on AGB stars in NGC 6752.

Acknowledgements. We are grateful to the anonymous referee for several suggestions that have improved the quality of this manuscript. P.G. acknowledges support from grant No. 2011- 5042 from the Swedish Research Council. S.F acknowledge the grant The New Milky Way from the Knut and Alice Wallenberg Foundation. P.G. and S.F. acknowledges support from the Swedish National Space Board. AS acknowledges support from MINECO (ESP201566134-R) and Generalitat de Catalunya (SGR2014-1458). L.C gratefully acknowledge support from the Australian Research Council (grants DP150100250, FT160100402). A.P.M. acknowledges support by the Australian Research Council through Discovery Early Career Researcher Award DE150101816.

\section{References}

Anderson, J., \& King, I. R. 2006, PSFs, Photometry, and Astronomy for the ACS/WFC, Tech. Rep.

Anderson, J., Bedin, L. R., Piotto, G., Yadav, R. S., \& Bellini, A. 2006, A\&A, 454, 1029

Árnadóttir, A. S., Feltzing, S., \& Lundström, I. 2010, A\&A, 521, A40

Bastian, N. 2015, ArXiv e-prints [arXiv: 1510.01330]

Bastian, N., Cabrera-Ziri, I., Davies, B., \& Larsen, S. S. 2013, MNRAS, 436, 2852

Brown, T. M., Cassisi, S., D’Antona, F., et al. 2016, ApJ, 822, 44

Calamida, A., Bono, G., Stetson, P. B., et al. 2007, ApJ, 670, 400

Campbell, S. W., Lattanzio, J. C., \& Elliott, L. M. 2006, Mem. Soc. Astron. Ital., 77,864

Campbell, S. W., D’Orazi, V., Yong, D., et al. 2013, Nature, 498, 198

4 The referee also pointed out that it is also possible that during the cooling, the H-burning shell present in the tiny residual envelope undergoes a He-flash along the WD sequence. A subsequent mixing episode then ensures that these stars end on the HB blue hook too. 
Carretta, E., Bragaglia, A., Gratton, R. G., Lucatello, S., \& Momany, Y. 2007, A\&A, 464, 927

Carretta, E., Bragaglia, A., Gratton, R., \& Lucatello, S. 2009a, A\&A, 505, 139

Carretta, E., Bragaglia, A., Gratton, R. G., et al. 2009b, A\&A, 505, 117

Carretta, E., Bragaglia, A., Gratton, R., D’Orazi D'Orazi, V., \& Lucatello, S. 2011a, A\&A, 535, A121

Carretta, E., Lucatello, S., Gratton, R. G., Bragaglia, A., \& D’Orazi, V. 2011b, A\&A, 533, A69

Casagrande, L., Silva Aguirre, V., Stello, D., et al. 2014, ApJ, 787, 110

Cassisi, S., Salaris, M., Pietrinferni, A., et al. 2008, ApJ, 672, L115

Cassisi, S., Salaris, M., Pietrinferni, A., Vink, J. S., \& Monelli, M. 2014, A\&A 571, A81

Castellani, V., Iannicola, G., Bono, G., et al. 2006, A\&A, 446, 569

D’Antona, F., Caloi, V., Montalbán, J., Ventura, P., \& Gratton, R. 2002, A\&A, 395,69

D’Antona, F., Vesperini, E., D’Ercole, A., et al. 2016, MNRAS, 458, 2122

Decressin, T., Meynet, G., Charbonnel, C., Prantzos, N., \& Ekström, S. 2007, A\&A, 464, 1029

Denissenkov, P. A., \& Hartwick, F. D. A. 2014, MNRAS, 437, L21

D'Orazi, V., Gratton, R. G., Angelou, G. C., et al. 2015, MNRAS, 449, 4038

Dorman, B., Rood, R. T., \& O’Connell, R. W. 1993, ApJ, 419, 596

Dotter, A., Ferguson, J. W., Conroy, C., et al. 2015, MNRAS, 446, 1641

García-Hernández, D. A., Mészáros, S., Monelli, M., et al. 2015, ApJ, 815, L4

Gratton, R. G., D’Orazi, V., Bragaglia, A., Carretta, E., \& Lucatello, S. 2010, A\&A, 522, A77

Gratton, R. G., Carretta, E., \& Bragaglia, A. 2012, A\&ARv, 20, 50

Greggio, L., \& Renzini, A. 1990, ApJ, 364, 35

Grundahl, F., Vandenberg, D. A., \& Andersen, M. I. 1998, ApJ, 500, L179

Grundahl, F., Catelan, M., Landsman, W. B., Stetson, P. B., \& Andersen, M. I. 1999, ApJ, 524, 242

Grundahl, F., Stetson, P. B., \& Andersen, M. I. 2002, A\&A, 395, 481

Gruyters, P., Nordlander, T., \& Korn, A. J. 2014, A\&A, 567, A72

Han, S.-I., Lee, Y.-W., Joo, S.-J., et al. 2009, ApJ, 707, L190

Ivans, I. I., Kraft, R. P., Sneden, C., et al. 2001, AJ, 122, 1438

Johnson, C. I., McDonald, I., Pilachowski, C. A., et al. 2015, AJ, 149, 71

Lapenna, E., Lardo, C., Mucciarelli, A., et al. 2016, ApJ, 826, L1

Lardo, C., Milone, A. P., Marino, A. F., et al. 2012, A\&A, 541, A141
Lardo, C., Salaris, M., Savino, A., et al. 2017, MNRAS, 466, 3507 MacLean, B. T., Campbell, S. W., De Silva, G. M., et al. 2016, MNRAS, 460 L69

Mallia, E. A. 1978, A\&A, 70, 115

Marino, A. F., Villanova, S., Piotto, G., et al. 2008, A\&A, 490, 625

Marino, A. F., Villanova, S., Milone, A. P., et al. 2011, ApJ, 730, L16

Marino, A. F., Milone, A. P., Przybilla, N., et al. 2014a, MNRAS, 437, 1609

Marino, A. F., Milone, A. P., Yong, D., et al. 2014b, MNRAS, 442, 3044

Marino, A. F., Milone, A. P., Karakas, A. I., et al. 2015, MNRAS, 450, 815

Massari, D., Lapenna, E., Bragaglia, A., et al. 2016, MNRAS, 458, 4162

Milone, A. P. 2015, MNRAS, 446, 1672

Milone, A. P., Bedin, L. R., Piotto, G., et al. 2008, ApJ, 673, 241

Milone, A. P., Marino, A. F., Piotto, G., et al. 2013, ApJ, 767, 120

Milone, A. P., Marino, A. F., Bedin, L. R., et al. 2014, MNRAS, 439, 1588

Milone, A. P., Marino, A. F., Piotto, G., et al. 2015a, MNRAS, 447, 927

Milone, A. P., Marino, A. F., Piotto, G., et al. 2015b, ApJ, 808, 51

Milone, A. P., Piotto, G., Renzini, A., et al. 2017, MNRAS, 464, 3636

Monelli, M., Milone, A. P., Stetson, P. B., et al. 2013, MNRAS, 431, 2126

Nardiello, D., Piotto, G., Milone, A. P., et al. 2015, MNRAS, 451, 312

Norris, J., Cottrell, P. L., Freeman, K. C., \& Da Costa, G. S. 1981, ApJ, 244, 205

Pancino, E., Rejkuba, M., Zoccali, M., \& Carrera, R. 2010, A\&A, 524, A44

Piotto, G., Milone, A. P., Bedin, L. R., et al. 2015, AJ, 149, 91

Renzini, A., D'Antona, F., Cassisi, S., et al. 2015, MNRAS, 454, 4197

Sbordone, L., Salaris, M., Weiss, A., \& Cassisi, S. 2011, A\&A, 534, A9

Smith, G. H., \& Norris, J. E. 1993, AJ, 105, 173

Strömgren, B. 1963, QJRAS, 4, 8

Sweigart, A. V., \& Gross, P. G. 1976, ApJS, 32, 367

Ventura, P., Caloi, V., D’Antona, F., et al. 2009, MNRAS, 399, 934

Villanova, S., Geisler, D., \& Piotto, G. 2010, ApJ, 722, L18

Villanova, S., Geisler, D., Piotto, G., \& Gratton, R. G. 2012, ApJ, 748, 62

Wang, Y., Primas, F., Charbonnel, C., et al. 2016, A\&A, 592, A66

Yong, D., Grundahl, F., Nissen, P. E., Jensen, H. R., \& Lambert, D. L. 2005, A\&A, 438, 875

Yong, D., Grundahl, F., Johnson, J. A., \& Asplund, M. 2008, ApJ, 684, 1159

Yong, D., Grundahl, F., D’ Antona, F., et al. 2009, ApJ, 695, L62

Yong, D., Meléndez, J., Grundahl, F., et al. 2013, MNRAS, 434, 3542

Yong, D., Grundahl, F., \& Norris, J. E. 2015, MNRAS, 446, 3319 


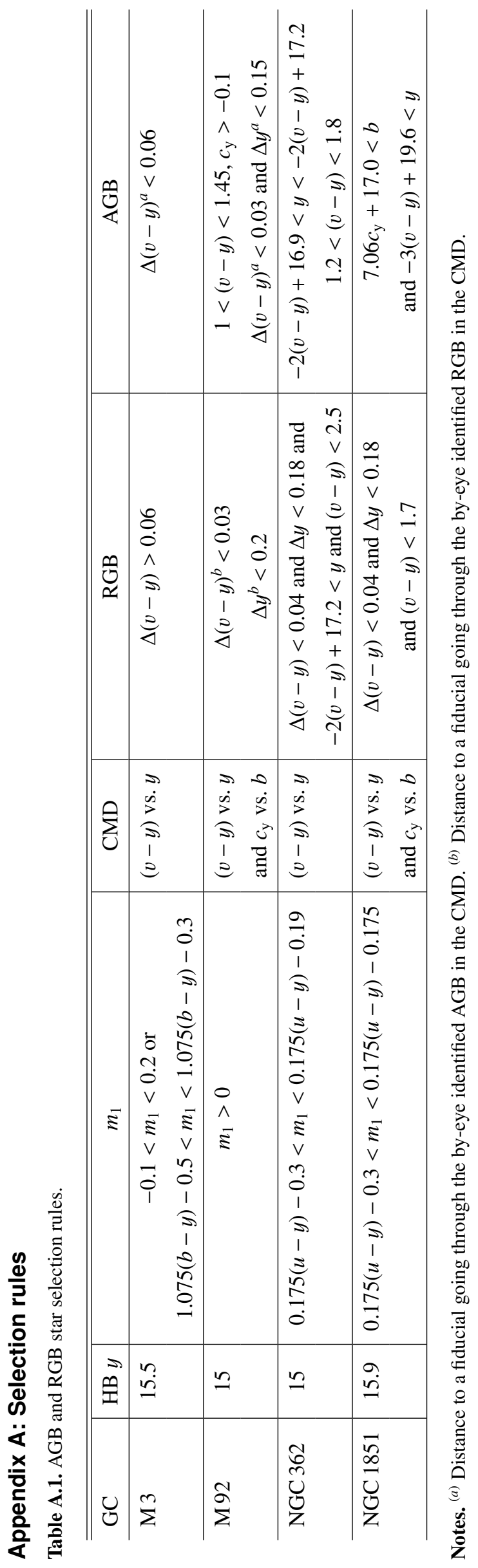


P. Gruyters et al.: First evidence of multiple populations along the AGB from Strömgren photometry

\section{Appendix B: Catalogue}

Table B.1. RGB and AGB catalogue based on the Grundahl photometry.

\begin{tabular}{lccccc}
\hline \hline GC & id & Ra & Dec & $y$ mag & Type \\
\hline NGC 362 & 813 & 15.834377 & -70.899370 & 14.92 & RGB \\
& 866 & 15.827797 & -70.898116 & 13.93 & RGB \\
& 1086 & 15.871138 & -70.892643 & 14.79 & RGB \\
& 1305 & 15.787733 & -70.889132 & 14.77 & RGB \\
& 2195 & 15.758909 & -70.875510 & 14.39 & RGB \\
\hline
\end{tabular}

Notes. Full table available at the CDS.

Table B.2. RGB and AGB catalogue for M3.

\begin{tabular}{ccccccc}
\hline \hline id & Ra & Dec & $u$ & $v$ & $b$ & $y$ \\
\hline RGB-1 & 205.7058305 & 28.3981320 & 17.244 & 16.015 & 15.182 & 14.589 \\
RGB-2 & 205.6732088 & 28.3189091 & 17.203 & 15.265 & 13.655 & 12.660 \\
RGB-3 & 205.6639402 & 28.4663573 & 16.837 & 15.110 & 13.873 & 13.048 \\
RGB-4 & 205.6480061 & 28.3328729 & 16.766 & 15.346 & 14.311 & 13.609 \\
RGB-5 & 205.6432670 & 28.4169339 & 17.182 & 16.012 & 15.239 & 14.635 \\
RGB-6 & 205.6338469 & 28.3839849 & 17.067 & 15.906 & 15.165 & 14.589 \\
\hline
\end{tabular}

Notes. Full table available at the CDS. 